\title{
Optimization of a new reactive force field for silver- based materials
}

Clement Dulong ${ }^{1}$, Bruno Madebene ${ }^{1}$, Susanna Monti ${ }^{2}$ and Johannes Richardi ${ }^{*}$

1 Sorbonne Université, CNRS, De la Molécule aux Nano-Objets: Réactivité, Interactions Spectroscopies, MONARIS, 75005, Paris France

2 CNR-ICCOM, Institute of Chemistry of Organometallic Compounds, via G. Moruzzi 1, I-56124 Pisa, Italy

3 Sorbonne Université, CNRS, Laboratoire de Chimie Théorique, LCT, 75005 Paris, France

SUPPORTING INFORMATION 


\begin{tabular}{|c|c|c|c|c|}
\hline & parameters & description & Ref. 24 & new \\
\hline 1 & r_0^sigma & Sigma bond covalent radius & 2.3644 & 2.4518 \\
\hline 2 & Val_i & Valency & 2.0000 & 2.0000 \\
\hline 3 & $\mathrm{n} / \mathrm{a}$ & Atomic mass & 63.5460 & 107.8682 \\
\hline 4 & r_vdW & van der Waals radius & 1.9896 & 2.0128 \\
\hline 5 & D_ij & van der Waals dissociation energy & 0.4000 & 0.3165 \\
\hline 6 & gamma_i & gammaEEM; EEM shielding & 0.4200 & 0.4200 \\
\hline 7 & r_0^pi & Pi bond covalent radius & 0.1000 & 0.1000 \\
\hline 8 & Val_i^e & Number of valence electrons & 1.0000 & 1.0000 \\
\hline 9 & alpha_ij & van der Waals parameter & 11.3629 & 11.0461 \\
\hline 10 & 1/gamma_w & van der Waals shielding & 100.0000 & 99.9077 \\
\hline 11 & Val_ $j^{\wedge}$ angle & Valency for $1,3-\mathrm{BO}$ correction & 1.0000 & 1.0000 \\
\hline 12 & p_ovun5 & Undercoordination energy & 0.0000 & 0.0000 \\
\hline 13 & $p_{-} \mathrm{i}^{\wedge} \mathrm{xel} 2$ & eReaxFF, atom type parameter & 0.0000 & 0.0000 \\
\hline 14 & chi_i & EEM electronegativity & 0.5000 & 1.0082 \\
\hline 15 & eta_i & EEM hardness & 6.0000 & 8.9305 \\
\hline 16 & $\mathrm{n} / \mathrm{a}$ & Donor or acceptor switch in H-bonds & 0.0000 & 0.0000 \\
\hline 17 & r_ $0^{\wedge} \mathrm{pi}$ & Double pi bond covalent radius & -1.0000 & -1.0000 \\
\hline 18 & p_lp2 & Lone pair energy & 0.0000 & 0.0000 \\
\hline 19 & $\mathrm{n} / \mathrm{a}$ & Atomic heat of formation & 80.7000 & 80.7000 \\
\hline 20 & p_boc4 & Bond order correction & 34.9923 & 35.750 \\
\hline 21 & p_boc3 & Bond order correction & 2.3973 & 5.2466 \\
\hline 22 & p_boc5 & Bond order correction & 0.0000 & 0.0000 \\
\hline 23 & $\mathrm{C}_{-} \mathrm{i}$ & Atomic softness cutoff parameter & 0.8563 & 0.8563 \\
\hline 24 & alpha, alpha_i & eReaxFF, constant, dependent on atom type & 0.0000 & 0.0000 \\
\hline 25 & p_ovun2 & Valence angle parameter & -5.1872 & -7.7845 \\
\hline 26 & p_val3 & Valence angle parameter & 3.8628 & 3.6694 \\
\hline 27 & beta, beta_i & eReaxFF, constant, dependent on atom type & 1.0000 & 1.0000 \\
\hline 28 & Val_i^’’boc & Number of lone pairs & 4.0000 & 4.0000 \\
\hline 29 & p_val5 & Valence angle parameter & 2.5791 & 2.5791 \\
\hline 30 & p_c1 & Inner wall vdW repulsion parameter & 0.0000 & 0.0000 \\
\hline 31 & p_c2 & Inner wall vdW repulsion parameter & 0.0000 & 0.0000 \\
\hline 32 & p_c3 & Inner wall vdW repulsion parameter & 0.0000 & 0.0000 \\
\hline 33 & $\mathrm{C}_{-} \mathrm{i}$ & Lg dispersion parameter & 0.0000 & 0.0000 \\
\hline 34 & R_eij & VdW Radius for Lg dispersion correction & 0.0000 & 0.0000 \\
\hline
\end{tabular}

Table S1 : Atomic parameters for silver: comparison between ReaxFF(lit) ${ }^{24}$ and the new force field. The optimized parameters a The optimized parameters are colored in red. 


\begin{tabular}{rclcc}
\hline & parameters & Description & Ref. 24 & new \\
\hline 1 & D_e ${ }^{\wedge}$ sigma & Sigma-bond dissociation energy & $\mathbf{5 9 . 5 1 7 9}$ & $\mathbf{6 7 . 7 8 0 5}$ \\
2 & D_e`pi & Pi-bond dissociation energy & 0.0000 & 0.0000 \\
3 & D_e ${ }^{\wedge}$ pipi & Double pi-bond dissociation energy & 0.0000 & 0.0000 \\
4 & p_be1 & Bond energy parameter & $\mathbf{- 0 . 2 3 7 9}$ & $\mathbf{- 0 . 4 8 9 1}$ \\
5 & p_bo5 & Double pi bond parameter & -0.2000 & -0.2000 \\
6 & Val’_i^boc & 1,3-Bond order correction & 0.0000 & 0.0000 \\
7 & p_bo6 & Double pi bond order & 16.0000 & 16.0000 \\
8 & p_ovun1 & Overcoordination penalty & $\mathbf{0 . 3 3 4 0}$ & $\mathbf{0 . 3 1 3 0}$ \\
9 & p_be2 & Bond energy parameter & $\mathbf{0 . 2 2 7 1}$ & $\mathbf{1 . 4 4 3 3}$ \\
10 & p_bo3 & Pi bond order parameter & -0.2000 & -0.2000 \\
11 & p_bo4 & Pi bond order parameter & 15.0000 & 15.0000 \\
12 & unused & n/a & 1.0000 & 1.0000 \\
13 & p_bo1 & Sigma bond order & $\mathbf{- 0 . 2 3 7 6}$ & $\mathbf{- 0 . 1 7 8 5}$ \\
14 & p_bo2 & Sigma bond order & $\mathbf{5 . 1 9 7 0}$ & $\mathbf{4 . 8 8 7 6}$ \\
15 & delta’i & Uncorrected BO overcoordination & 0.0000 & 0.0000 \\
16 & p_ij^xel1 & e ReaxFF param & 0.0000 & 0.0000 \\
\hline
\end{tabular}

Table S2 : Bond parameters for silver : comparison between ReaxFF(lit) and the new force field. The optimized parameters are displayed in red letters. 


\begin{tabular}{rclc}
\hline & parameters & description & new \\
\hline 1 & D_e ${ }^{\wedge}$ sigma & Sigma-bond dissociation energy & 129.5847 \\
2 & D_e ${ }^{\wedge}$ pi & Pi-bond dissociation energy & 0.0000 \\
3 & D_e ${ }^{\wedge}$ pipi & Double pi-bond dissociation energy & 0.0000 \\
4 & p_be1 & Bond energy parameter & -0.6766 \\
5 & p_bo5 & Double pi bond parameter & -0.2000 \\
6 & Val'_i^boc & 1,3-Bond order correction & 0.0000 \\
7 & p_bo6 & Double pi bond order & 16.0000 \\
8 & p_ovun1 & Overcoordination penalty & -0.0198 \\
9 & p_be2 & Bond energy parameter & 0.1321 \\
10 & p_bo3 & Pi bond order parameter & -0.2000 \\
11 & p_bo4 & Pi bond order parameter & 15.0000 \\
12 & unused & n/a & 1.0000 \\
13 & p_bo1 & Sigma bond order & -0.4592 \\
14 & p_bo2 & Sigma bond order & 5.2259 \\
15 & delta'_i & Uncorrected BO overcoordination & 0.0000 \\
16 & p_ij`xel1 & e ReaxFF param & 0.0000 \\
\hline
\end{tabular}

Table S3: Bond parameters for Ag-S of the new force field. The optimized parameters are displayed in red letters.

\begin{tabular}{lllc}
\hline \multicolumn{1}{c}{ parameters } & description & new \\
\hline 1 & D_ij & VdW energy & 0.2440 \\
2 & r_vdW & VdW radius & 2.0564 \\
3 & alpha_ij & VdW parameter & 11.8537 \\
4 & r_0^sigma & Sigma bond length & 2.1389 \\
5 & r_0^pi & Pi bond length & -1.0000 \\
6 & r_0^pipi & PiPi bond length & -1.0000 \\
7 & C_i, C_lg,ij & Lg dispersion parameter & -1.0000 \\
\hline
\end{tabular}

Table S4: Off-diagonal parameters for Ag-S of the new force field. The optimized parameters are displayed in red letters. 


\begin{tabular}{lllc}
\hline \multicolumn{1}{c}{ parameters } & Description & new \\
\hline 1 & Theta_0,0 & $180^{\circ}$-(equilibrium angle) & 107.9986 \\
2 & p_val1 & Valence angle parameter & -21.7445 \\
3 & p_val2 & Valence angle parameter & 15.6034 \\
4 & p_coa1 & Valence conjugation & 0.0000 \\
5 & p_val7 & Undercoordination & 2.4513 \\
6 & p_pen1 & Penalty energy & 0.0000 \\
7 & p_val4 & Valence angle parameter & 1.9208 \\
\hline
\end{tabular}

Table S5: Torsion parameters for S-Ag-Ag of the new force field. The optimized parameters are displayed in red letters.

\begin{tabular}{lllc}
\hline parameters & Description & new \\
\hline 1 & Theta_0,0 & $180^{\circ}$-(equilibrium angle) & 53.9262 \\
2 & p_val1 & Valence angle parameter & 53.8120 \\
3 & p_val2 & Valence angle parameter & 20.9984 \\
4 & p_coa1 & Valence conjugation & 0.0000 \\
5 & p_val7 & Undercoordination & 4.9781 \\
6 & p_pen1 & Penalty energy & 0.0000 \\
7 & p_val4 & Valence angle parameter & 2.1841 \\
\hline
\end{tabular}

Table S6: Torsion parameters for Ag-S-Ag of the new force field. The optimized parameters are displayed in red letters. 


\begin{tabular}{|c|c|c|c|c|c|}
\hline Isomers & potentials & $\mathrm{E}_{\mathrm{rel}} / \mathrm{kcal} / \mathrm{mol}$ & d(Au-S) /Å & $\mathbf{d}(\mathbf{A u}-\mathbf{A u}) / \AA$ & 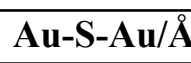 \\
\hline \multirow{4}{*}{$\mathbf{E E}_{\text {edge }}$} & Järvi & 0 & 2.58 & 3.02 & 72 \\
\hline & Bae & 0 & 2.48 & 3.16 & 79 \\
\hline & Monti & 0 & 2.48 & 3.03 & 75 \\
\hline & DFT & 0 & 2.37 & 3.25 & 87 \\
\hline \multirow{4}{*}{ VE } & Järvi & 15 & 2.59 & 3.01 & 71 \\
\hline & Bae & 1 & $\mathrm{E}: 2.49 \mathrm{~V}: 2.47$ & 3.14 & 78 \\
\hline & Monti & 9 & $\mathrm{E}: 2.48 \mathrm{~V}: 2.49$ & 3.01 & 75 \\
\hline & DFT & 10 & $\mathrm{E}: 2.43 \mathrm{~V}: 2.39$ & 2.90 & 74 \\
\hline \multirow{4}{*}{$\mathbf{V}$} & Järvi & 56 & 2.54 & - & - \\
\hline & Bae & -30 & 2.42 & - & - \\
\hline & Monti & 99 & 2.28 & - & - \\
\hline & DFT & 23 & 2.28 & - & - \\
\hline \multirow{4}{*}{$\mathbf{F E}_{\text {down }}$} & Järvi & 76 & $\mathrm{E}: 2.67 \mathrm{~F}: 2.63$ & 2.97 & 68 \\
\hline & Bae & 51 & $\mathrm{E}: 2.50 \mathrm{~F}: 2.44$ & 3.68 & 97 \\
\hline & Monti & 129 & $\mathrm{E}: 2.66 \mathrm{~F}: 2.55$ & 3.01 & 71 \\
\hline & DFT & 39 & $\mathrm{E}: 2.46 \mathrm{~F}: 2.37$ & 3.05 & 78 \\
\hline \multirow{4}{*}{$\mathbf{E} \mathbf{E}_{\text {down }}$} & Järvi & 43 & $\mathrm{E}: 2.61 \mathrm{~V}: 2.71$ & 3.07 & 72 \\
\hline & Bae & -2 & no bond $\mathrm{Au}-\mathrm{S}$ & 2.89 & - \\
\hline & Monti & 114 & $\mathrm{E}: 2.43 \mathrm{~V}: 2.38$ & 3.02 & 77 \\
\hline & DFT & 66 & $\mathrm{E}: 2.50 \mathrm{~V}: 3.02$ & 2.87 & 70 \\
\hline
\end{tabular}

Table S7: Relative energies (kJ / mol), S-S and Au-Au interatomic distances ( $\AA$ ) and AuS-Au angles $\left({ }^{\circ}\right)$ for each cluster optimized with three ReaxFF available (Järvi, Bae, Monti) as well as the DFT reference values. 


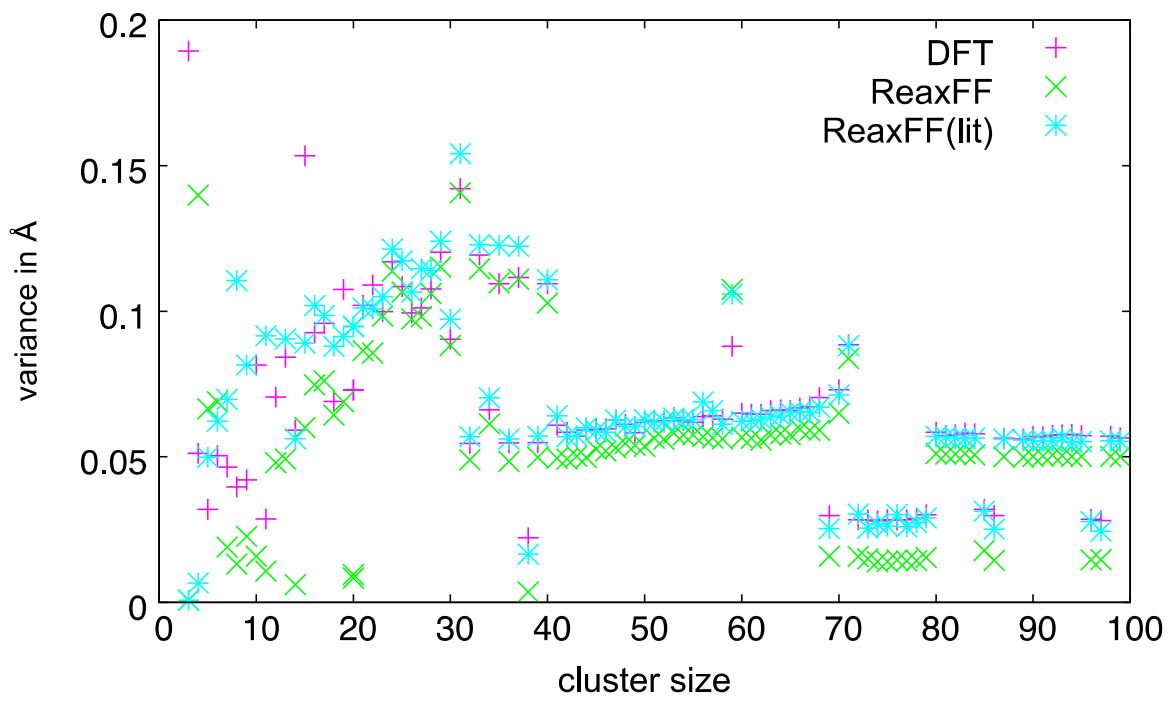

Figure S1: Standard deviation of the first-neighbor distances between silver atoms as a function of the cluster size (from 2 to 99 silver atoms) for DFT, the published reactive force field (ReaxFF (lit)), ${ }^{24}$ and the one developed here (ReaxFF). 


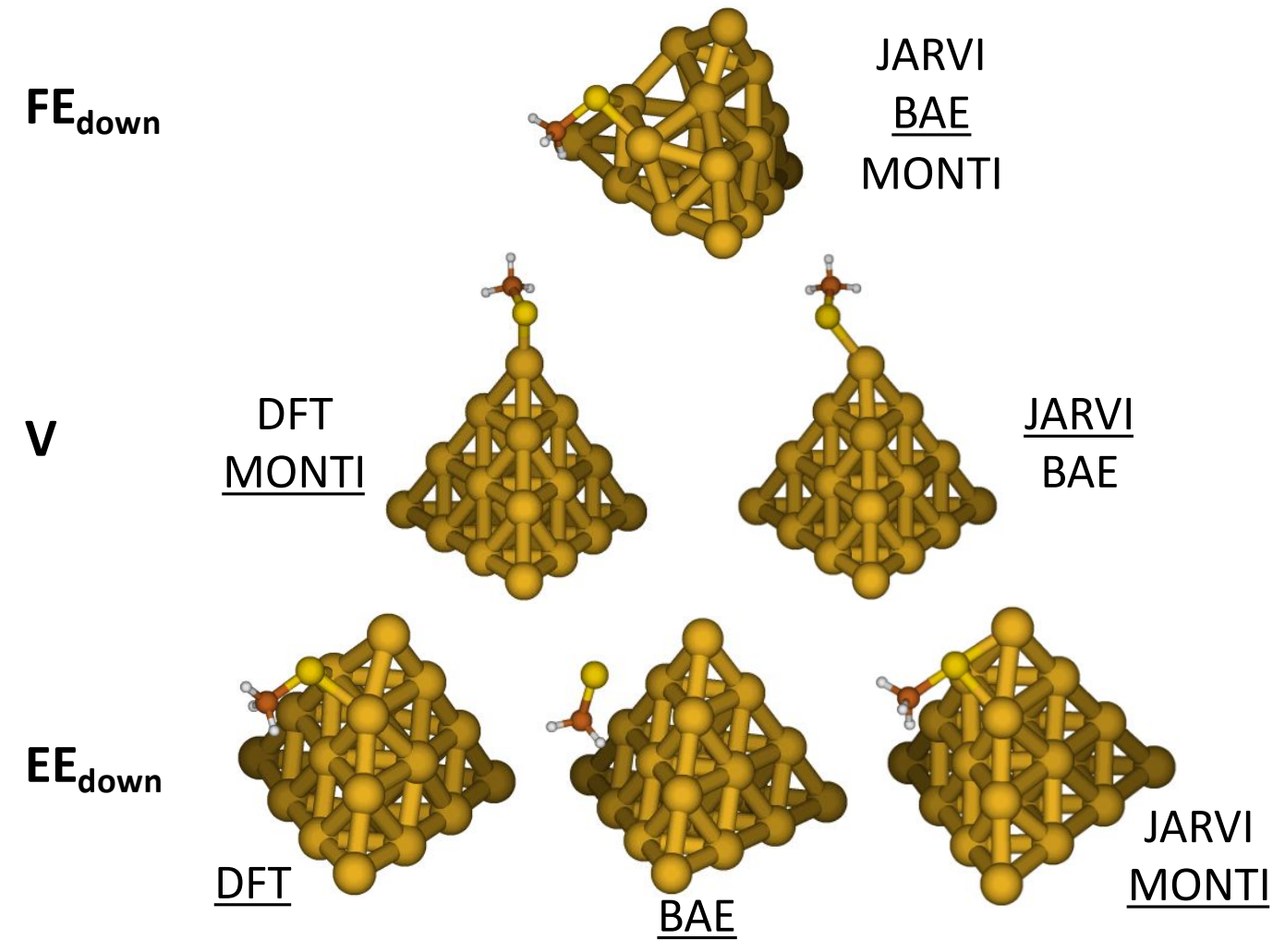

Figure S2: Examples of the different geometries obtained by ReaxFF and DFT for the $\mathrm{FE}_{\text {down }}$ (first line), $\mathrm{V}$ (second line) and $\mathrm{EE}_{\text {down }}$ (third line) isomers for MeS-Au $\mathbf{u}_{20}$. The shown structure corresponds to the underlined potential. 
Methanethiolate on $\mathrm{Ag}(111)$, new ReaxFF $100 \mathrm{~K}$ $100 \mathrm{~K}$ (without alkane chain)

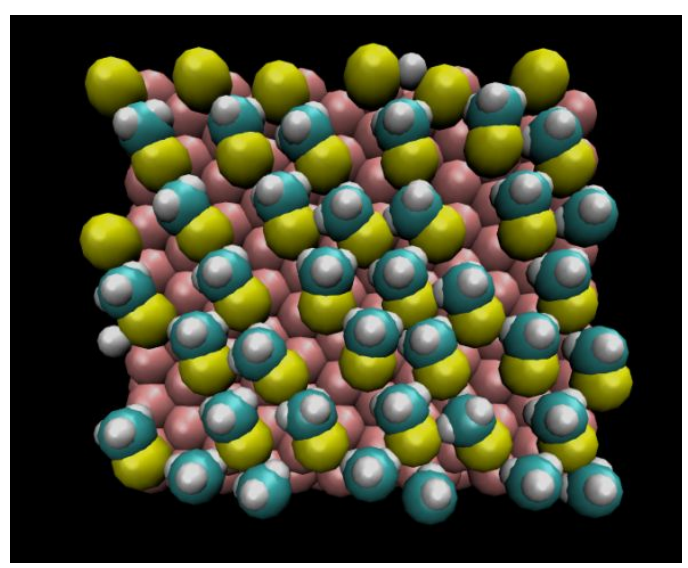

$300 \mathrm{~K}$

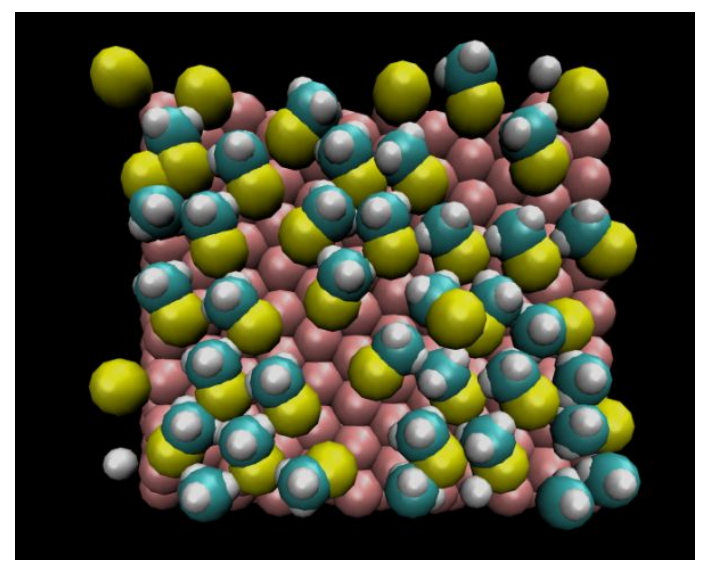

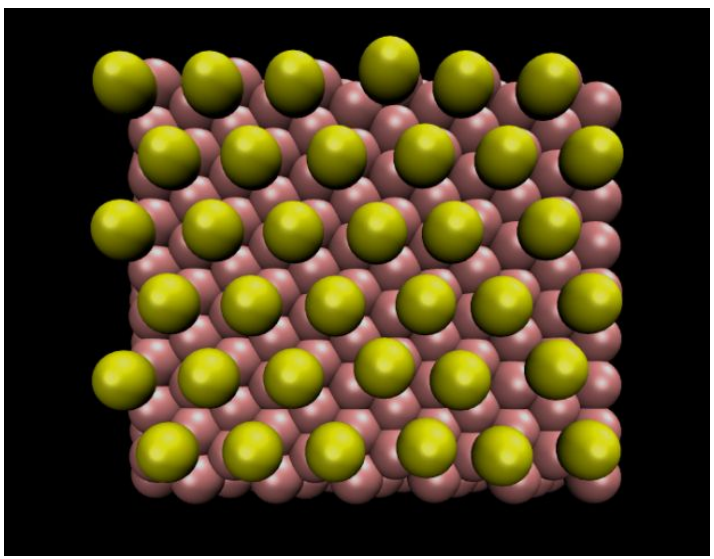

$300 \mathrm{~K}$ (without alkane chain)

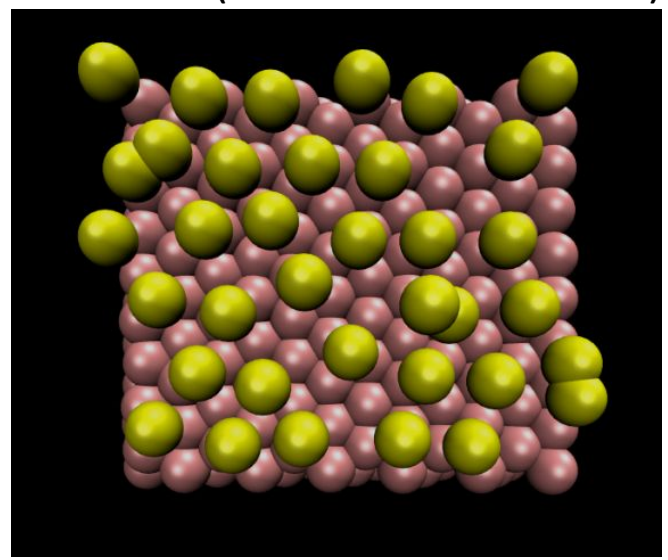

Figure S3: Snapshots of simulations of a SAM of methanethiolate on silver using the new ReaxFF at 100 and $300 \mathrm{~K}$. On the right, the alkane chains have been removed to see the assembly of sulfur atoms. 
Hexanethiolate on $\mathrm{Ag}(111)$, new ReaxFF

$100 \mathrm{~K}$

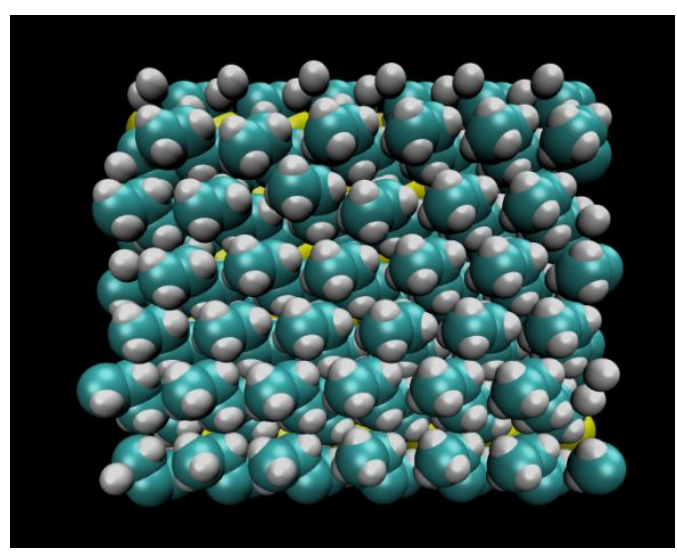

$300 \mathrm{~K}$

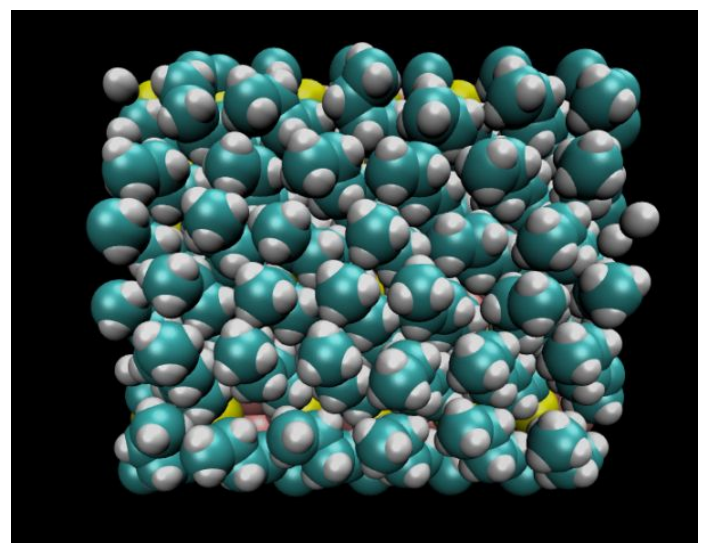

$100 \mathrm{~K}$ (without alkane chain)

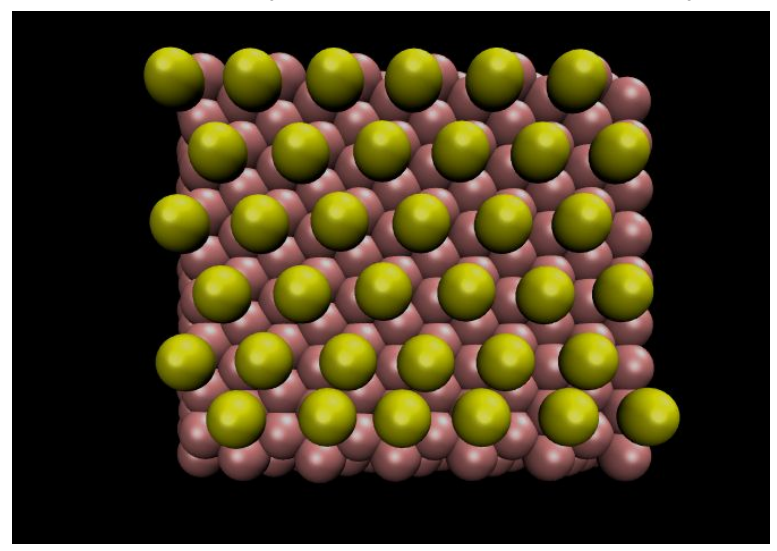

$300 \mathrm{~K}$ (without alkane chain)

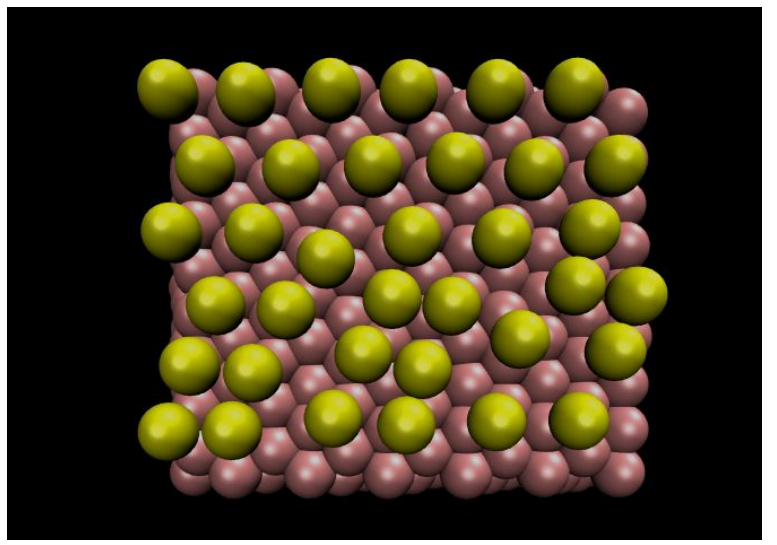

Figure S4: Snapshots of simulations of a SAM of hexanethiolate on silver using the new ReaxFF at 100 and $300 \mathrm{~K}$. On the right, the alkane chains have been removed to see the assembly of sulfur atoms. 
Methanethiolate on Au(111), ReaxFF by Järvi et al. $100 \mathrm{~K}$ $100 \mathrm{~K}$ (without alkane chain)
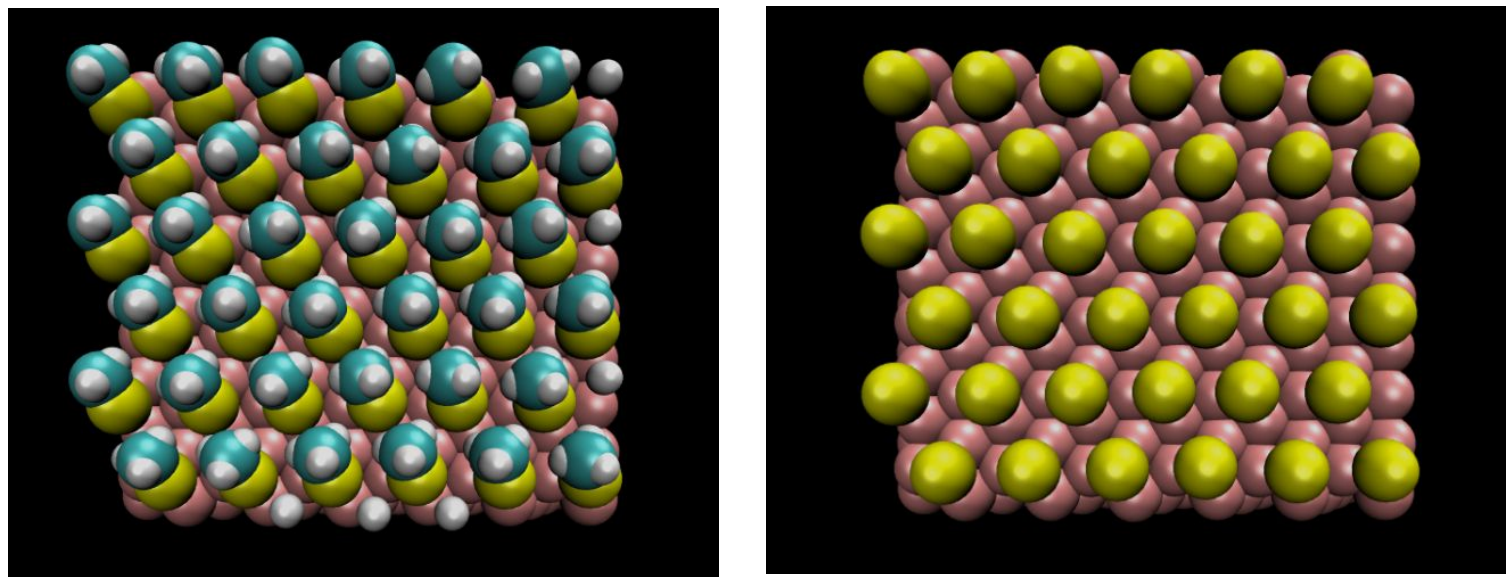

$300 \mathrm{~K}$

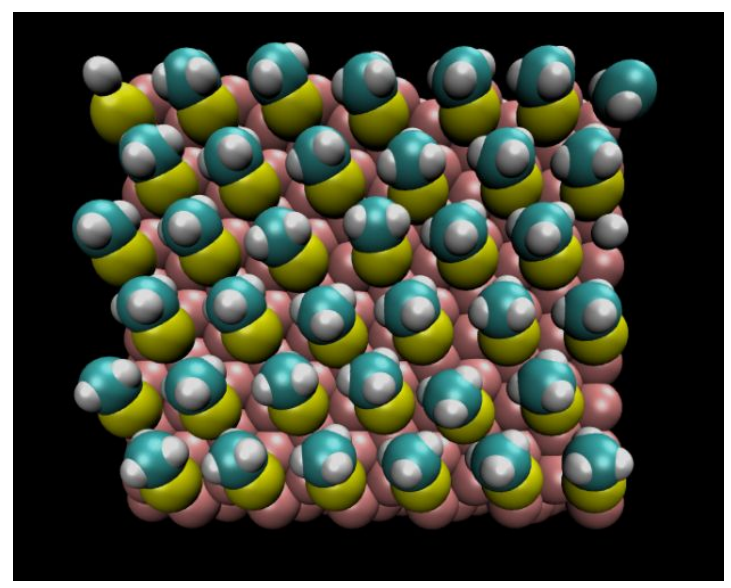

$300 \mathrm{~K}$ (without alkane chain)

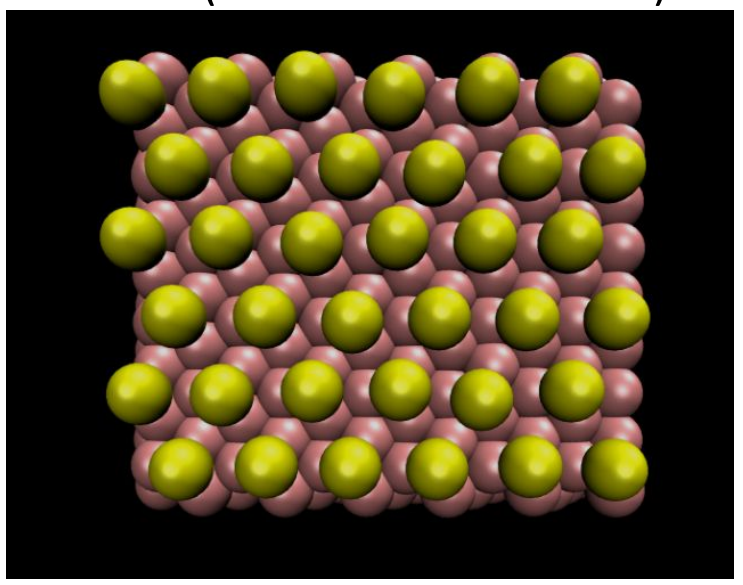

Figure S5: Snapshots of simulations of a SAM of methanethiolate on gold using the ReaxFF proposed by Järvi et al. at 100 and $300 \mathrm{~K}$. On the right, the alkane chains have been removed to see the assembly of sulfur atoms. 
Hexanethiolate on $\mathrm{Au}(111)$, ReaxFF by Järvi et al.

$100 \mathrm{~K}$

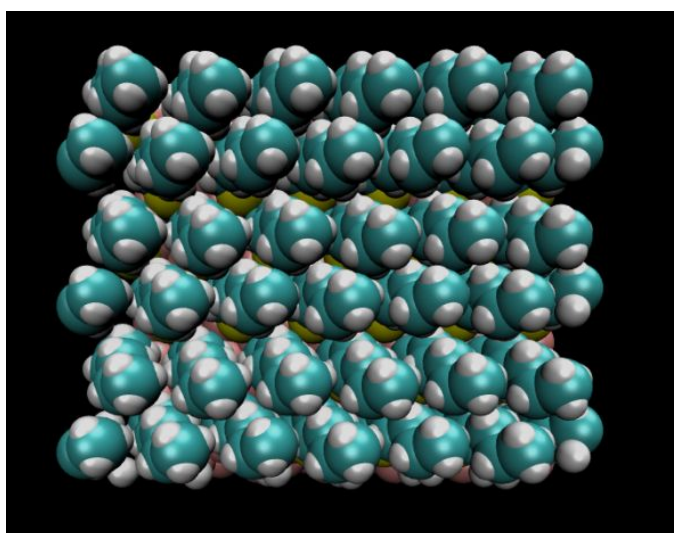

$300 \mathrm{~K}$

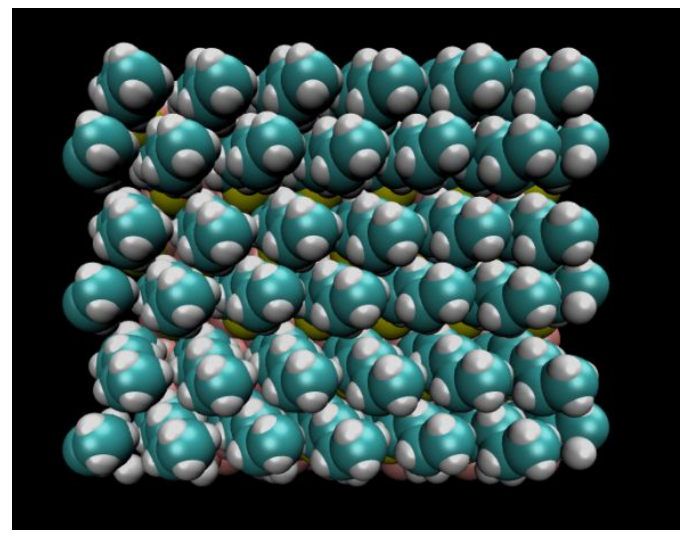

$100 \mathrm{~K}$ (without alkane chain)

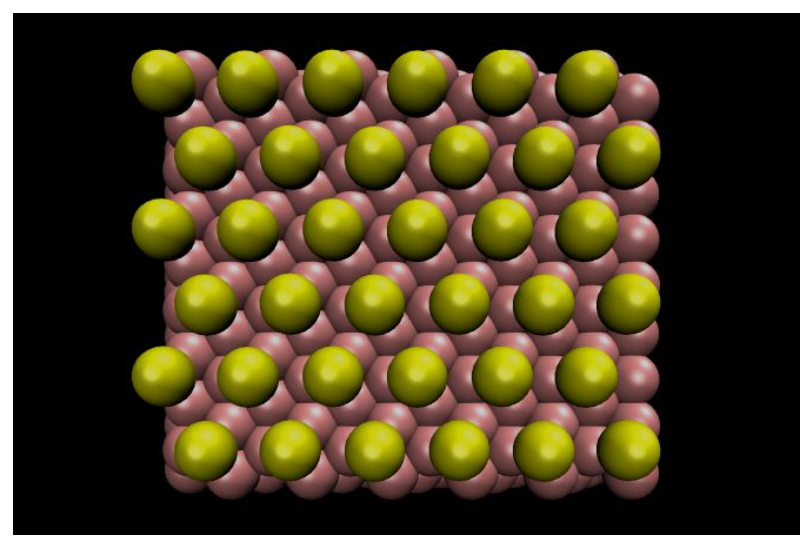

$300 \mathrm{~K}$ (without alkane chain)

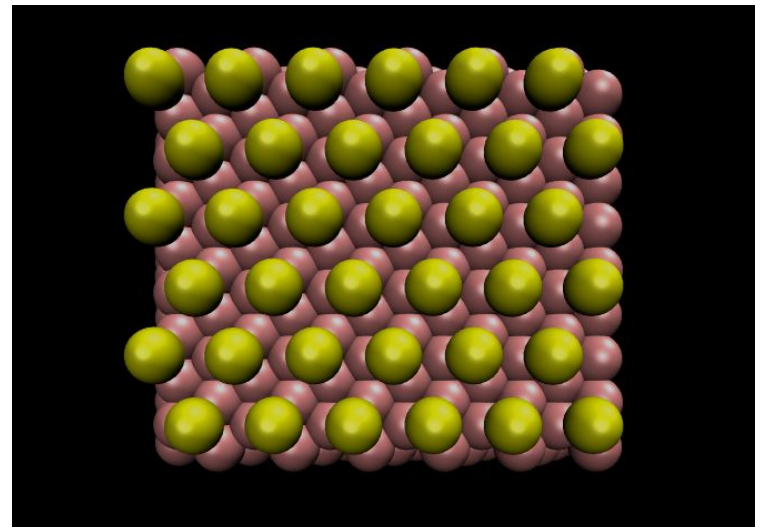

Figure S6: Snapshots of simulations of a SAM of hexanethiolate on gold using the ReaxFF proposed by Järvi et al. at 100 and $300 \mathrm{~K}$. On the right, the alkane chains have been removed to see the assembly of sulfur atoms. 
Methanethiolate on $\mathrm{Au}(111)$, ReaxFF by Monti et al. $100 \mathrm{~K}$ $100 \mathrm{~K}$ (without alkane chain)
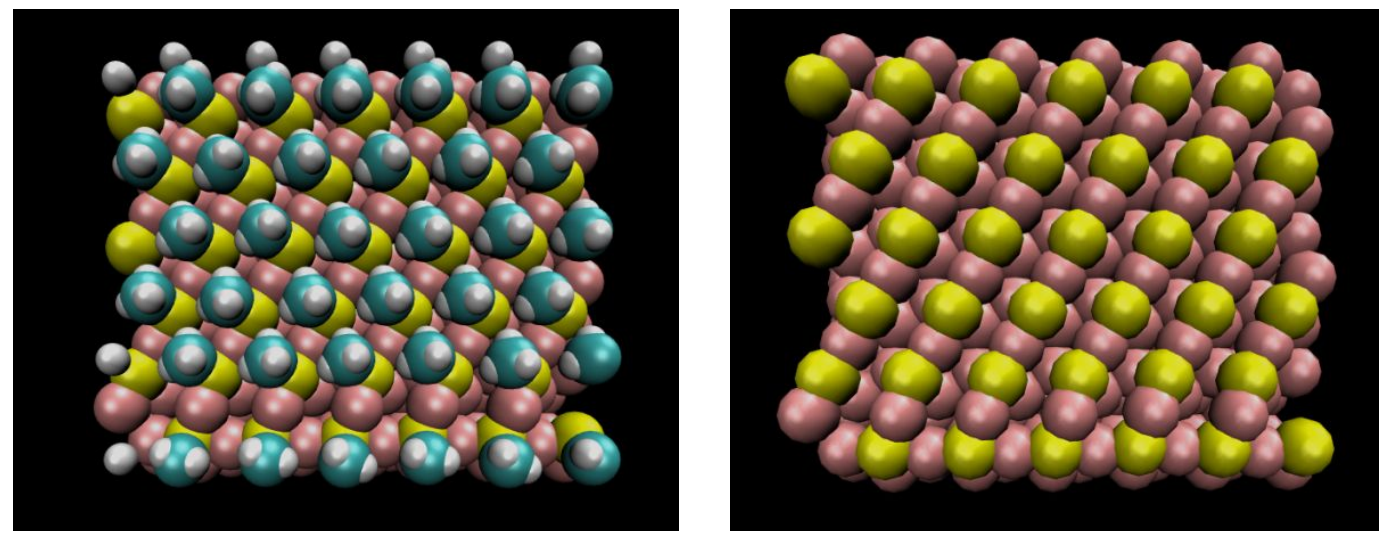

$300 \mathrm{~K}$

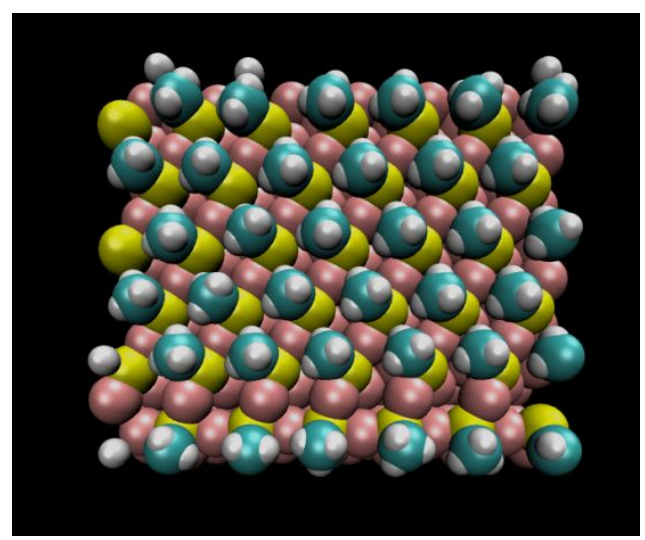

$300 \mathrm{~K}$ (without alkane chain)

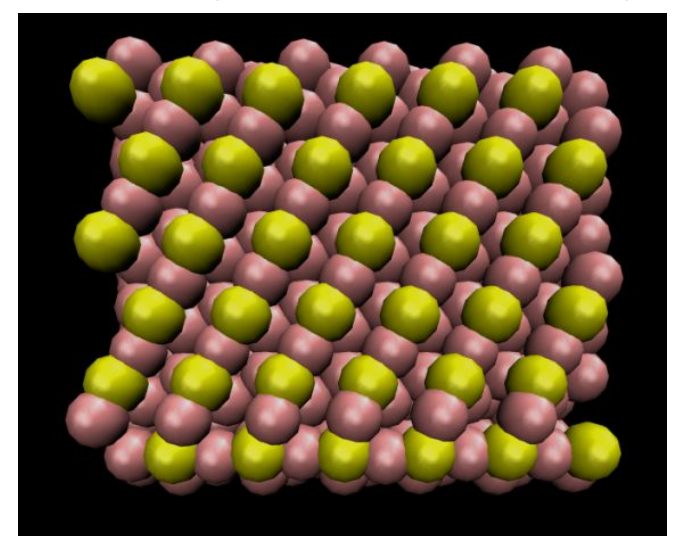

Figure S7: Snapshots of simulations of a SAM of methanethiolate on gold using the ReaxFF proposed by Monti et al. at 100 and $300 \mathrm{~K}$. On the right, the alkane chains have been removed to see the assembly of sulfur atoms. 
Hexanethiolate on $\mathrm{Au}(111)$, ReaxFF by Monti et al.

$100 \mathrm{~K}$

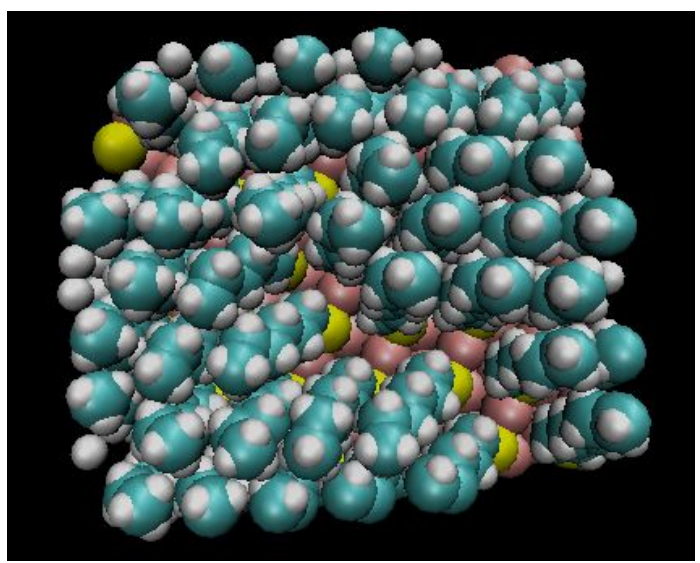

$300 \mathrm{~K}$

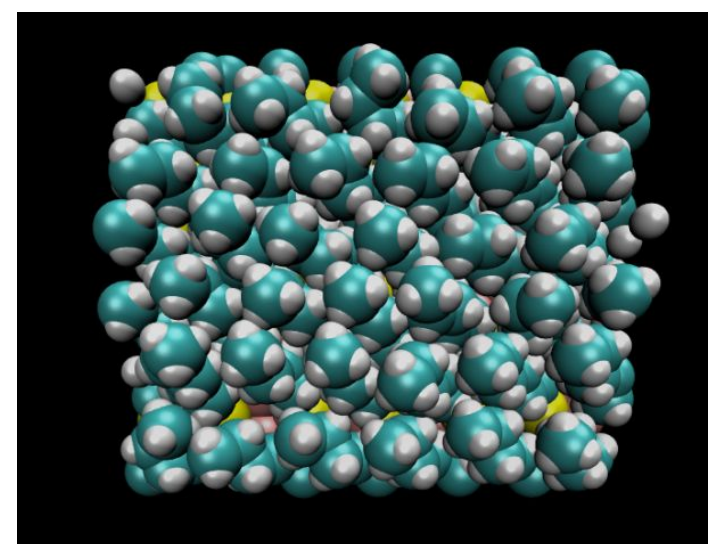

$100 \mathrm{~K}$ (without alkane chain)

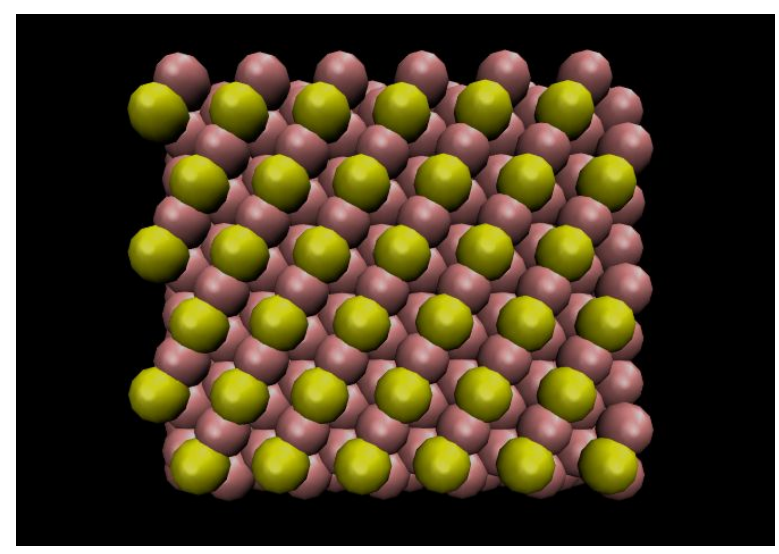

$300 \mathrm{~K}$ (without alkane chain)

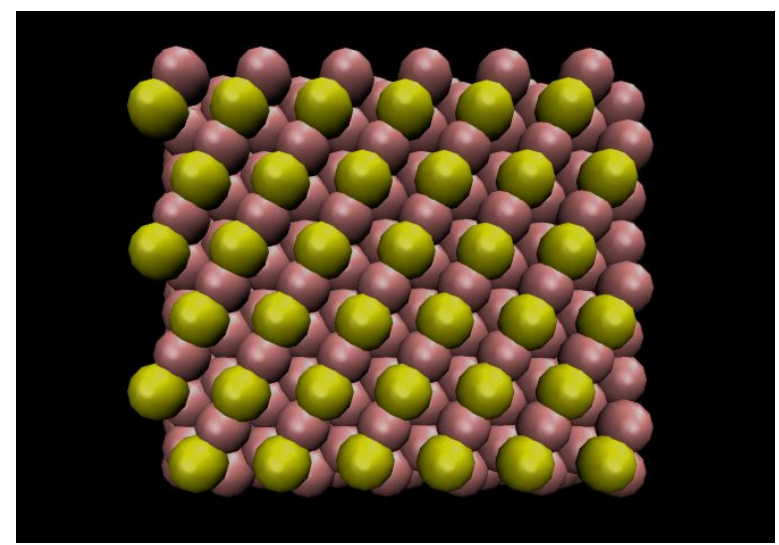

Figure S8: Snapshots of simulations of a SAM of hexanethiolate on gold using the two ReaxFF proposed by Monti et al. at $300 \mathrm{~K}$. On the right, the alkane chains have been removed to see the assembly of sulfur atoms. 
Hexanethiolate on Au(111), ReaxFF by Bae and Aikins

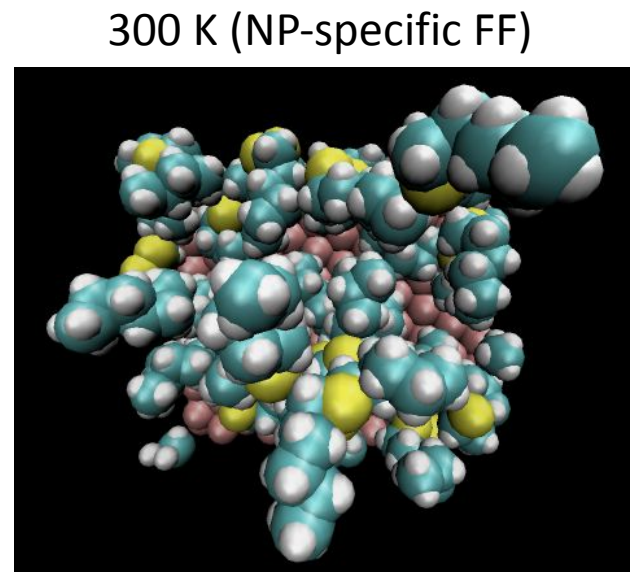

$300 \mathrm{~K}$ (NP-specific FF, without alkane chain)

$300 \mathrm{~K}$ (FF of table 1)

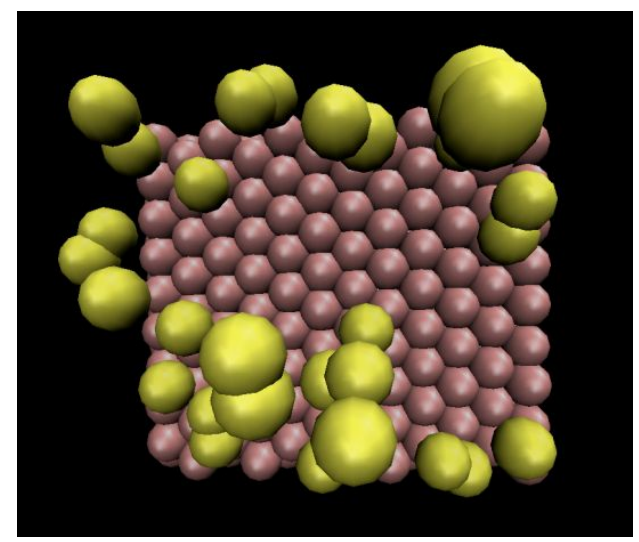

$300 \mathrm{~K}$ (FF of table 1 , without alkane chain)
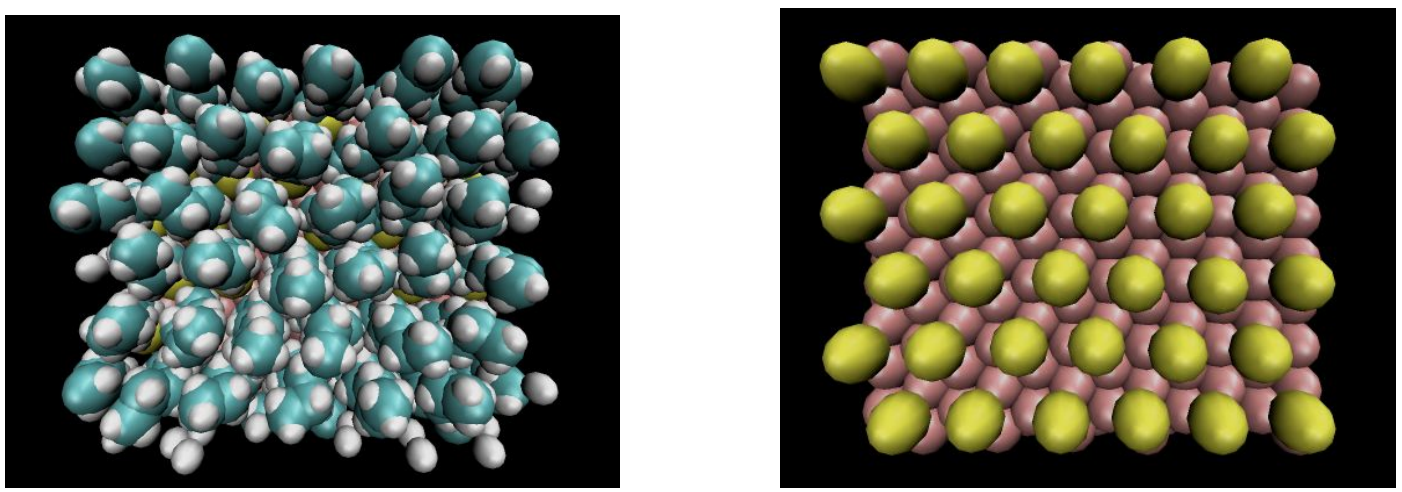

Figure S9: Snapshots of simulations of a SAM of hexanthiolate on gold using the two ReaxFF proposed by Bae et al. at $300 \mathrm{~K}$. 